\title{
Construction of a barley gene regulatory network for phenolic metabolism using mutants and near-isogenic lines
}

\author{
Olesya Shoeva \\ Kurchatov Genomics Center, \\ ICG SB RAS, Novosibirsk, Russia \\ olesya_ter@bionet.nsc.ru \\ Tatjana Kukoeva \\ Kurchatov Genomics Center, \\ ICG SB RAS, Novosibirsk, Russia \\ kukoeva@bionet.nsc.ru \\ Mats Hansson \\ Lund University, Lund, Sweden \\ mats.hansson@biol.lu.se
}

\author{
Ksenia Strygina \\ VIR, St. Petersburg, Russia \\ k.strygina@vir.nw.ru \\ Elena Gordeeva \\ ICG SB RAS, Novosibirsk, Russia \\ elgordeeva@bionet.nsc.ru \\ Elena Khlestkina \\ ICG SB RAS, Novosibirsk, Russia \\ VIR, St. Petersburg, Russia \\ khlest@bionet.nsc.ru
}

\author{
Anastasiia Glagoleva \\ Kurchatov Genomics Center, \\ ICG SB RAS, Novosibirsk, Russia \\ glagoleva@bionet.nsc.ru \\ Shakhira Zakhrabekova \\ Lund University, Lund, Sweden \\ shakhira.zakhrabekova@biol.lu.se
}

\begin{abstract}
In the present study, the components of the gene regulatory network governing the anthocyanin and proanthocyanidin biosynthesis in barely grain and leaf sheath were identified based on comparative mapping and transcription analysis approaches. The regulatory relationships between the genes were established using unique barley anthocyanin/proanthocyanidin mutants and near-isogenic lines.
\end{abstract}

Keywords - anthocyanins, proanthocyanidins, structural and regulatory genes, regulatory loops, Hordeum vulgare $L$.

\section{Motivation and aim}

Phenolic compounds are plant secondary metabolites with important functions in plant physiology and adaptation [1]. They are synthesized in various parts of the plant through phenylpropanoid biosynthesis pathway and different branches of flavonoid biosynthesis pathway. In barley (Hordeum vulgare L.), the application of the grain is dependent on the phenolic composition. For example, proanthocyanidins (also known as condensed tannins) are undesirable in barley cultivars used in malt production as they cause chill haze reducing beer quality [2]. In contrast, anthocyanins that can accumulate in aleurone layer and pericarp and cause blue and purple color of grain, respectively, are promising functional food ingredients and are desirable in cultivars for human consumption [3]. More information is required to fully understand how to manage the content of phenolic compounds in the barley grain. Two groups of genes control the biosynthesis of phenolic compounds: structural genes that encode enzymes of the pathway, and regulatory genes that encode MYB, bHLH or WD40 transcriptional factors (TFs). The TFs form complexes that activate transcription of the structural genes in a tissue-specific manner [4].

The aim of the current study was to identify the components of the gene regulatory networks underlining synthesis of phenolic compounds in barley grains and to study regulatory relationships between them in the course of synthesis of anthocyanins and proanthocyanidins.

\section{Methods}

A set of barley mutants with lesion in anthocyanin and/or proanthocyanidin biosynthesis and Bowman near-isogenic lines (NILs) differing in anthocyanin pigmentation of grain and leaf sheath were used in the study. The barley lines were obtained from NordGen (Alnarp, Sweden). Nucleotide sequences of the candidate genes for the Ant1, Ant2, Ant 5, Ant13 loci were determined by Sanger sequencing of the corresponding mutants, NILs and their parental cultivars. Expression of the anthocyanin biosynthesis genes in grain and leaf sheath was explored by qRT-PCR.

\section{Results}

The regulatory function of the Ant1 (mapped to chromosome 7HS), Ant2 (2HL) and Ant13 (6HL) loci on anthocyanin biosynthesis were suggested based on the previous studies of the anthocyanin/proanthocyanidin-less barley mutants [5]. Comparative mapping data allowed us to assign molecular functions to the loci. The Ant1 gene encodes a MYB-like TF, required for the activation of expression of the anthocyanin biosynthesis structural genes in leaf sheath [6,7], while the Ant2 gene encodes a bHLH-type TF that is required together with Ant1 for the activation of the anthocyanin biosynthesis in grain pericarp [8]. Ant13 encodes a WD40 TF. SNPs leading to stop codons and amino acid substitutions were identified in the ant 13 mutants. In the case of the Ant1 and Ant2 genes differences in promoter regions were identified and were assumed to prevent expression of the recessive alleles of these genes in non-colored grain pericarp. Bowman and its purple-grained NIL with dominant alleles of the both Ant1 and Ant2 genes were used to create new lines with different combinations of the dominant and recessive alleles of these genes [8]. Expression analysis of the structural genes in the obtained NILs revealed that the transcription of the structural gene Ans that encodes anthocyanidin synthase is strongly dependent on the dominant alleles of the both Ant1 and Ant2 genes. As the analysis of the anthocyanin-less mutants demonstrated that the Ant1, Ant2 and Ant5 loci controlled together the reaction of conversion of leucoanthocyanidins into anthocyanidins catalyzed by ANS [5], the Ans gene was suggested as a candidate gene for the Ant5 locus. Sequencing of the Ans gene in a set of ant5 mutants and parental cultivars revealed the SNPs causing stop codons and amino acid substitutions destroying the protein function.

The regulatory relationships between the Ant1 and Ant2 genes were established. Transcription of Ant2 was upregulated in the presence of a dominant allele of Ant1 and transcription of Antl was increased in the presence of a dominant allele of Ant2. The data suggested existence of a 
positive regulatory loop between the two genes. The present study is the first report on interaction between the anthocyanins regulatory genes in monocot plant species.

\section{Acknowledgment}

The study was supported by RFBR grant №16-34-60052, the Kurchatov Genomics Center of ICG № 075-15-20191662, the Swedish Institute Visby Programme 25896/2018, the Swedish Research Council (VR 2018-05117), and the Swedish Research Council for Environment, Agricultural Sciences and Spatial Planning (FORMAS 2018-01026).

\section{References}

[1] Babenko L.M. (2019). Phenolic compounds in plants: biogenesis and functions. The Ukrainian Biochemical Journal. 91: 5-18.

[2] von Wettstein D. (2007). From analysis of mutants to genetic engineering. Annual Review of Plant Biology. 109: 232-249.
[3] Zhu F. (2018). Anthocyanins in cereals: composition and health effects. Food Research International. 109: 232-249.

[4] Hichri I. et al. (2011). Recent advances in the transcriptional regulation of the flavonoid biosynthetic pathway, Journal of Experimental Botany. 62: 2465-2483.

[5] Jende-Strid B. (1993). Genetic control of flavonoid biosynthesis in barley. Hereditas 119: 187-204.

[6] Shoeva et al. (2015). Barley Ant1 is a homolog of maize $C 1$ and its product is part of the regulatory machinery governing anthocyanin synthesis in the leaf sheath. Plant Breeding 134: 400-405.

[7] Zakhrabekova et al. (2015). Genetic linkage facilitates cloning of Ert$m$ regulating plant architecture in barley and identified a strong candidate of Ant1 involved in anthocyanin biosynthesis. Plant Molecular Biology. 88: 609-626.

[8] Gordeeva et al. (2019). Purple-grained barley (Hordeum vulgare L.): marker-assisted development of NILs for investigating peculiarities of the anthocyanin biosynthesis regulatory network. BMC Plant Biology 19: 52 . 